\title{
Splitting of Surface Plasmon Frequencies of Metal Particles in a Nematic Liquid Crystal
}

\author{
Sung Yong Park and D. Stroud \\ Department of Physics, The Ohio State University, Columbus, Ohio 43210
}

\begin{abstract}
We calculate the effective dielectric function for a suspension of small metallic particles immersed in a nematic liquid crystal (NLC) host. For a random suspension of such particles in the dilute limit, we calculate the effective dielectric tensor exactly and show that the surface plasmon (SP)resonance of such particles splits into two resonances, polarized parallel and perpendicular to the NLC director. At higher concentrations, we calculate this splitting using a generalized Maxwell-Garnett approximation, which can also be applied to a small metal particle coated with NLC. To confirm the accuracy of the MGA for NLC-coated spheres, we also use the Discrete Dipole Approximation. The calculated splitting is comparable to that observed in recent experiments on NLC-coated small metal particles.
\end{abstract}


An important goal in optics is to prepare materials with optical properties easily controllable by external parameters such as temperature or electromagnetic fields. Nematic liquid crystals (NLC's) are useful materials of this kind [1-4]: the optical axis of an NLC, known as the director, can be rotated by a weak applied electric field $\mathcal{E}$, thereby altering its transmission and reflection coefficients. Several authors have suggested that such control might be achieved by incorporating NLC's into composite media which are structured on a scale comparable to the wavelength [5-11].

Recently, there have been new optical applications of nanoparticles on a scale smaller than the wavelength [12]. Among the applications are nanolenses [13], plasmonic nanoantennas [14], and light energy transfer through linear chains of nanoparticles [15-17]. Thus, it would be of great interest if the use of NLC's to control light propagation could be extended to systems with structure on this scale. Indeed, a recent experiment has shown that the optical properties of NLC-coated metal nanoparticles can be controlled by a dc electric field [18].

In this Letter, as a first step towards a theoretical treatment of such systems, we present an exact calculation of the optical properties of a dilute suspension of metallic nanoparticles in an NLC host. We find that the usual surface plasmon (SP) resonance of the metal nanoparticles splits into two resonances, polarized parallel and perpendicular to the NLC director. In addition, we develop a Maxwell-Garnett approximation (MGA) which is suitable either for higher concentrations of particles in an NLC host or for a single nanoparticle coated with an NLC. We also use the discrete dipole approximation (DDA) to confirm the accuracy of the MGA for NLC-coated spheres. For typical NLC parameters, we find that the calculated SP splitting for coated spheres is large enough to be experimentally observable in both absorption and transmission, and, indeed, is of a magnitude comparable to that found in experiments [18].

First, we consider the optical properties of a dilute suspension of spherical metallic nanoparticles in an NLC host, as illustrated schematically in Fig. 1 (a). The metal particles are assumed to have a complex but isotropic dielectric function $\epsilon_{m}(\omega)$, which we take simply 
to be of the Drude form,

$$
\epsilon_{m}(\omega)=1-\omega_{p}^{2} /[\omega(\omega+i / \tau)]
$$

where $\omega_{p}$ is the plasma frequency and $\tau$ is a characteristic relaxation time. The NLC host is taken to be a uniaxial dielectric with dielectric tensor $\tilde{\epsilon}_{N L C}$ having principal components $\epsilon_{\perp} \equiv n_{\perp}^{2}, \epsilon_{\perp}$, and $\epsilon_{\|} \equiv n_{\|}^{2}$, assumed real and frequency-independent. If the metal particle radius is small compared to either its skin depth or the wavelength $\lambda$, the composite optical properties are well described by a complex effective dielectric tensor, $\tilde{\epsilon}_{e}(\omega)$. We also assume that the NLC is homogeneous, with principal axes which point in the same directions at every point in the space it occupies. (The limitations of this assumption are discussed briefly below.) With these approximations, $\tilde{\epsilon}_{e}(\omega)$ is also a tensor with principal components $\epsilon_{e, \perp}(\omega)$, $\epsilon_{e, \perp}(\omega)$, and $\epsilon_{e, \|}(\omega)$.

We calculate $\tilde{\epsilon}_{e}(\omega)$ in the limit that the volume fraction of inclusions $p \ll 1$. In actuality, $\epsilon_{e}$ for this geometry has been calculated in Ref. [19] for the formally analogous case of conducting particle in a host with an anisotropic d. c. conductivity. The result for the dielectric function is

$$
\tilde{\epsilon}_{e}(\omega)=\tilde{\epsilon}_{1}+p[\tilde{1}-\delta \tilde{\epsilon} \tilde{\Gamma}]^{-1} \delta \tilde{\epsilon}
$$

where $\delta \tilde{\epsilon}=\tilde{\epsilon_{2}}-\tilde{\epsilon_{1}}$ is the difference between the diagonal dielectric tensors $\epsilon_{2}$ and $\epsilon_{1}$ of the inclusion and the host, and $\tilde{\Gamma}$ is a $3 \times 3$ "depolarization tensor" which is diagonal in the same frame of reference as $\tilde{\epsilon}_{1}$. In the case of Fig. 1(a), $\tilde{\epsilon}_{1}$ corresponds to $\tilde{\epsilon}_{N L C}$ and $\tilde{\epsilon}_{2}$ to $\epsilon_{m} \tilde{1}$, where $\tilde{1}$ is a $3 \times 3$ unit matrix. Equation (2) is a matrix equation for $\tilde{\epsilon}_{e}$ but because both $\tilde{\Gamma}$ and $\delta \tilde{\epsilon}$ are simultaneously diagonalizable, it is also diagonal and reduces to three separate algebraic equations for the three components of $\tilde{\epsilon}_{e}$. If the metallic inclusions are spherical particles, then $\tilde{\Gamma}$ has principal components $\Gamma_{\perp}, \Gamma_{\perp}$, and $\Gamma_{\|}$, where

$$
\Gamma_{\|}=-\left(1-\sqrt{1-g} \sin ^{-1} \sqrt{g} / \sqrt{g}\right) /\left(\epsilon_{\|} g\right),
$$

and 


$$
\Gamma_{\perp}=-\frac{1}{2}\left(\Gamma_{\|}+\sin ^{-1} \sqrt{g} / \sqrt{g \epsilon_{\|} \epsilon_{\perp}}\right)
$$

where $g=1-\epsilon_{\perp} / \epsilon_{\|}$.

The positions $\omega_{\alpha}(\alpha=\|$ or $\perp)$ of the two peaks can be obtained analytically from the equation

$$
1-\Gamma_{\alpha} \delta \epsilon_{\alpha}=0
$$

where $\alpha=\|$ or $\perp$. For a Drude dielectric function in the limit $\omega_{p} \tau \rightarrow \infty$, this equation reduces to

$$
\omega_{0, \alpha}^{2} / \omega_{p}^{2}=-\Gamma_{\alpha} /\left[1-\left(1-\epsilon_{\alpha}\right) \Gamma_{\alpha}\right]
$$

where $\Gamma_{\alpha}$ is given by Eqs. (3) and (4). To present illustrative results, we assume that the host is the NLC known as E7, which has principal indices of refraction $n_{\perp}=\sqrt{\epsilon_{\perp}}=1.52, n_{\|}=$ $\sqrt{\epsilon_{\|}}=1.75$, as used in the experiments in Ref. [18]. For these $n_{\perp}$ and $n_{\|}, \omega_{0, \alpha} / \omega_{p}=0.4068$ and 0.4022 . Here the difference is a small but observable amount, and is robust against small changes of either index of refraction. It is of interest to compare the calculated splitting with that observed in the experiments. For $p=0.01$, our calculated splitting between the $\|$ and $\perp \mathrm{SP}$ frequencies is about 1\%. By comparison, that observed in Ref. [18] appears to be around $3.4 \%$.

At higher concentrations, $\tilde{\epsilon}_{e}$ can only be computed approximately, e. g., using the Maxwell-Garnett approximation (MGA). The MGA is believed to be best suited to a geometry in which the inclusions are preferentially surrounded by the host (NLC, in this case). If the director $\hat{n}$ of the NLC is independent of position, then the MGA takes the form $\tilde{\epsilon}_{M G A}=\epsilon_{2}+p[\tilde{1}-(1-p) \tilde{\Gamma} \delta \tilde{\epsilon}]^{-1} \delta \tilde{\epsilon}$.

Next, we consider the optical properties of a dilute suspension of coated particles. The coating is assumed to consist of a thin layer of NLC. The geometry in this case is shown schematically in Fig. 1(b). We may calculate the effective dielectric function of this suspension in two stages. First, we calculate the dielectric function $\tilde{\epsilon}_{\text {coat }}$ of the coated particle using 
the MGA, and assuming that the director $\hat{n}$ in the coating is uniformly oriented. Thus, we take

$$
\tilde{\epsilon}_{\text {coat }}=\tilde{\epsilon}_{N L C}+p^{\prime}\left[\tilde{1}-\left(1-p^{\prime}\right) \tilde{\Gamma} \delta \tilde{\epsilon}\right]^{-1} \delta \tilde{\epsilon}
$$

where $s$ is the thickness of the coating, $a$ is the radius of the included metallic particle, $p^{\prime}=a^{3} /(a+s)^{3}$ is the volume fraction of metal in the coated particle, and $\delta \tilde{\epsilon}=\epsilon_{m} \tilde{1}-\tilde{\epsilon}_{N L C}$. In the second stage, we assume that a volume fraction $p \ll 1$ of coated particles is embedded in an air host, and calculate the effective dielectric tensor $\epsilon_{e}$ of this suspension using eq. (2). To carry out this latter calculation, we take $\tilde{\epsilon}_{1}=\tilde{1}, \delta \tilde{\epsilon}=\tilde{\epsilon}_{\text {coat }}-\tilde{1}$, and use $\tilde{\Gamma}=-\tilde{1} / 3$, the depolarization tensor for a spherical particle in an isotropic host of unit dielectric constant. The resulting $\tilde{\epsilon}_{e}$ is diagonal with nonzero components

$$
\epsilon_{e, \mu}=1+p t_{\mu}
$$

where

$$
t_{\mu}=\frac{\epsilon_{\text {coat }, \mu}-1}{1+(1 / 3)\left(\epsilon_{\text {coat }, \mu}-1\right)},
$$

and the subscript $\mu=\|$ or $\perp$ denotes a component in the direction parallel or perpendicular to $\hat{n}$. By analogy with Eq. (5), we can determine the positions $\omega_{\mu}$ of the two peaks analytically from the equation

$$
1+(1 / 3)\left(\epsilon_{\text {coat }, \mu}-1\right)=0
$$

where $\mu=\|$ or $\perp$.

We have used Eq. (10) to obtain the SP resonance frequencies of a dilute suspension of NLC-coated metal particles. We consider two different angles between the director and the polarization of incident light, namely $0^{\circ}$ and $90^{\circ}$. We use the Drude dielectric function of Eq. (1) for $\epsilon_{m}(\omega)$, in the limit $\omega_{p} \tau \rightarrow \infty$, and the dielectric tensor of E7 for that of the coating. The resulting frequencies of the SP resonances are shown in Fig. 2 as full and dashed lines.

To see how well this MGA approach works for a dilute suspension of coated metal particles, we have compared the results to DDA calculations of the normalized peak position 
of scattered amplitude [20]. In the DDA, the scattering object is represented as a collection of point dipoles on a simple cubic grid of $n^{3}$ points, with $n$ as large as 120; we increase $n$ until the peak position of scattered amplitude converges. The magnitudes of the point dipoles are chosen to reproduce the scattering properties of the original medium [21]. We use the Drude dielectric function of Eq. (1) for $\epsilon_{m}(\omega)$, with $\omega_{p} \tau=7.7$, and the dielectric tensor of E7 for that of the coating. The peak positions, calculated in the DDA and interpreted as SP frequencies, are shown in Fig. 2 as open squares and diamonds. These calculations show that, for $p^{\prime} \gtrsim 0.5$, the MGA and DDA results are very close. For $a=30 \mathrm{~nm}$ this result means that for $s \lesssim 8 n m$, the MGA result is reasonable. Thus, the Maxwell-Garnett approach is fairly accurate for the optical response of nanoparticles with a thin NLC coating, when the director is uniform in the coating.

Finally, we discuss the limitation of our present calculations. In a plausible NLC layer thickness range, the calculated splitting in Fig. 2 between the SP frequencies for light polarized parallel and perpendicular to $\hat{n}$ is $1 \sim 2 \%$, roughly comparable to experiment [18]. However, to make a detailed comparison to experiment, we would need to relax some of the assumptions made in the present calculations; the assumption that the NLC is homogeneous, near the surface of a colloidal particle, may be very difficult to achieve experimentally, since in real experimental situations, the NLC director is substantially perturbed near the surface of a colloidal particle. The characteristics of the surface of the particle, which are referred to as anchoring conditions, can yield various nontrivial configurations of the nematic liquid crystal [22]; these anchoring conditions will vary with the experimental preparation [23]. Thus, a more quantitatively accurate theory of the optical properties of suspensions should include these effects, though the present approach should provide a qualitatively reasonable first step. Calculations which take into account these complications will be presented elsewhere [24].

This work has been supported by NSF grant DMR 01-04987. We would like to thank Dr. S. Maier for useful conversations. Numerical calculations in this paper were carried out using the facilities of the Ohio Supercomputer Center. 


\section{REFERENCES}

[1] P. G. de Gennes and J. Prost, The Physics of Liquid Crystals (Clarendon Press, Oxford, U. K., 1993).

[2] S. Chandrasekhar, Liquid Crystals (Cambridge U. P., Cambridge, U. K., 1992).

[3] L. M. Blinov and V. G. Chigrinov, Electro-Optic Effects in Liquid Crystal Materials (Springer-Verlag, New York, 1994).

[4] P. M. Chaikin and T. C. Lubensky, Principles of Condensed Matter Physics (Cambridge University Press, Cambridge, U. K., 1995), pp. 58-71.

[5] K. Busch and S. John, Phys. Rev. Lett. 83, 967 (1999).

[6] I. H. H. Zabel and D. Stroud, Phys. Rev. B 48, 5004 (1993).

[7] Z.-Y. Li, J. Wang, and B.-Y. Gu, Phys. Rev. B 58, 3721 (1998).

[8] C.-S. Kee, H. Lim, Y.-K. Ha, J.-E. Kim, and H. Y. Park, Phys. Rev. B 64, 085114 (2001).

[9] D. Kang, J. E. Maclennan, N. A. Clark, A. A. Zakhidov, and R. H. Baughman, Phys. Rev. Lett. 86, 4052 (2001).

[10] Y. Shimoda, M. Ozaki, and K. Yoshino, Appl. Phys. Lett. 79, 3627 (2001).

[11] S. W. Leonard, J.P. Mondia, H. M. van Driel, O. Toadar, S. John, K. Busch, A. Birner, U. Gösele, and V. Lehmann, Phys. Rev. B 61, R2389 (2000).

[12] See, e. g. "http://surfaceplasmonoptics.org".

[13] K. Li, M. I. Stockman, and D. J. Bergman, Phys. Rev. Lett. 91, 227402 (2003).

[14] D. A. Genov, A. K. Sarychev, V. M. Shalaev, and A. Wei, Nano Lett. 4, 153 (2004).

[15] M. Quinten, A. Leitner, J. R. Krenn, and F. R. Ausenegg, Opt. Lett. 23, 1331 (1998). 
[16] S. A. Maier, M. L. Brongersma, P. G. Kik, S. Meltzer, A. A. G. Requicha, and H. A. Atwater, Adv. Mater. 13, 1501 (2001).

[17] S. A. Maier, P. G. Kik, H. A. Atwater, S. Meltzer, E. Harel, B. G. Koel, and A. A. G. Requicha, Nature Materials 2, 229 (2003); J. R. Krenn, Nature Materials 2, 210 (2003).

[18] J. Müller, C. Sönnichsen, H. von Poschinger, G. von Plessen, T. A. Klar, and J. Feldmann, Appl. Phys. Lett. 81, 171 (2002)

[19] D. Stroud and F. P. Pan, Phys. Rev. B 13, 1434 (1976).

[20] E. M. Purcell and C. R. Pennypacker, Astrophys. J. 186, 705 (1973); J. J. Goodman, B. T. Draine, and P. J. Flatau, Opt. Lett. 16, 1198 (1991).

[21] For an anisotropic medium with diagonal dielectric tensor $\tilde{\epsilon}$, the polarizability tensor of these point dipoles also becomes a diagonal tensor when we use the "Lattice Dispersion Relation". See, e. g., B. T. Draine and P. J. Flatau, J. Opt. Soc. Am. A 11, 1491 (1994).

[22] T. C. Lubensky, D. Pettey, N. Currier, and H. Stark, Phys. Rev. E 57, 610 (1998); P. Poulin and D.A. Weitz, Phys. Rev. E 57, 626 (1998); H. Stark, Phys. Rep. 351, 387 (2001) and references therein; R. D. Kamien and T. D. Powers, Liq. Cryst. 23, 213 (1997); D. W. Allender, G. P. Crawford, and J. W. Doane, Phys. Rev. Lett. 67, 1442 (1991).

[23] P. Poulin, H. Stark, T.C. Lubensky, and D.A. Weitz, Science 275, 1770 (1997); J. Chen, P.J. Bos, H. Vithana, and D.L. Johnson, Appl. Phys. Lett. 67, 2588 (1995); V.K. Gupta and N.L. Abbott, Science 276, 1533 (1997).

[24] S. Y. Park and D. Stroud (unpublished). 


\section{FIGURE CAPTIONS}

FIGURE 1. Schematic diagram of the geometries we consider here. (a) metal nanoparticle embedded in an NLC host. (b) NLC coated metal nanoparticle in air host.

FIGURE 2. Calculated surface plasmon frequencies $\omega_{0, \|}\left(p^{\prime}\right) / \omega_{0}$ and $\omega_{0, \perp}\left(p^{\prime}\right) / \omega_{0}$, corresponding to incident light polarized parallel and perpendicular to the NLC director, for metal nanoparticles coated with NLC and embedded in an air host, plotted as a function of volume fraction $p^{\prime}$ of the coated particle which is composed of metal. The coating is assumed to be the NLC E7, which has indices of refraction $n_{\|}=1.75, n_{\perp}=1.52$. The metal particles are taken to have the Drude dielectric function, and $\omega_{0} \equiv \omega_{0}\left(p^{\prime}=1\right)$. Full and dashed lines: MGA; squares and circles: DDA. 


\section{FIGURES}

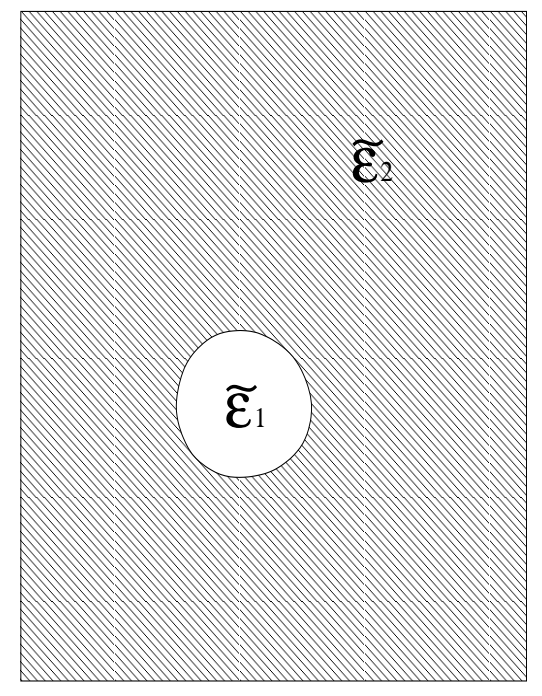

(a)

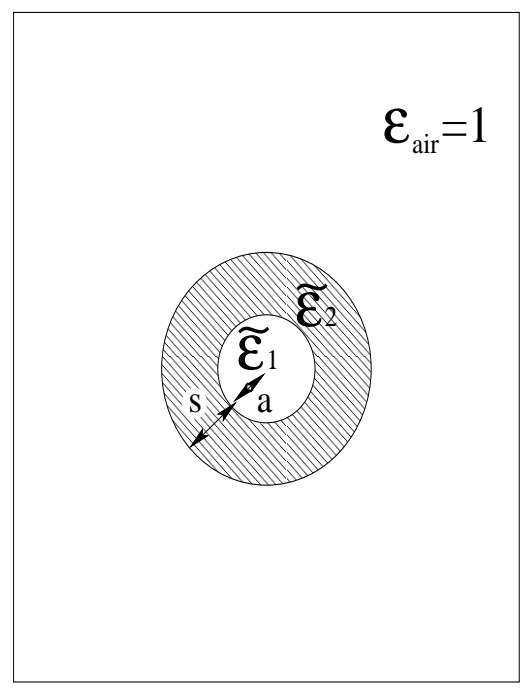

(b)

FIG. 1. 


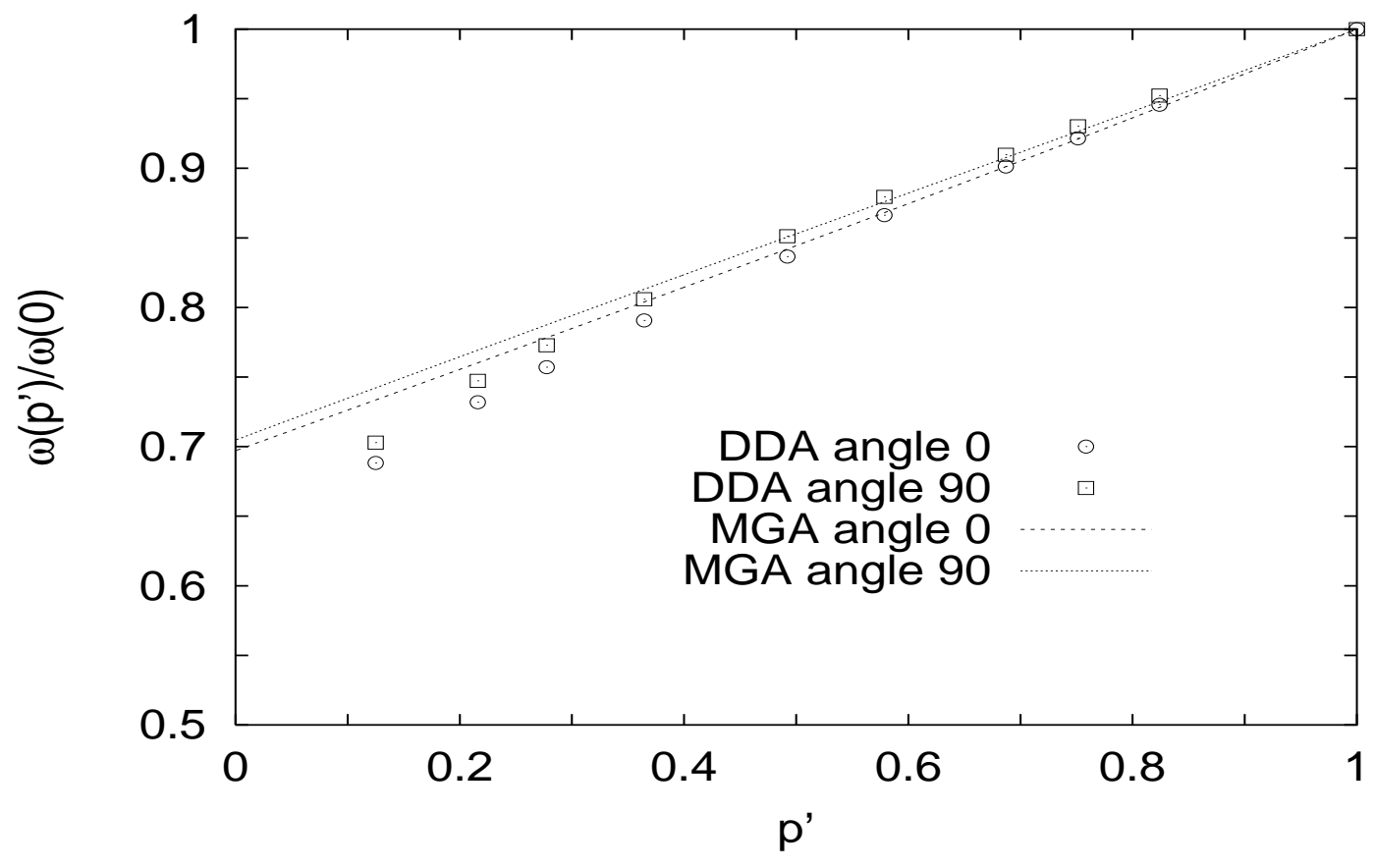

FIG. 2. 